\title{
Estudio Anatómico del Fascículo Accesorio del Músculo Flexor Largo del Pulgar y su Relación con el Nervio Interóseo Anterior en Individuos Brasileños
}

\author{
Anatomical Study of the Accessory Head of the Flexor Pollicis Longus Muscle \\ and its Relationship to the Anterior Interosseous Nerve in Brazilian Individuals
}

Riveros A."**; Olave, E.**** \& Sousa-Rodrigues, C.*****

RIVEROS, A.; OLAVE, E. \& SOUSA-RODRIGUES, C. Estudio anatómico del fascículo accesorio del músculo flexor largo del pulgar y su relación con el nervio interóseo anterior en individuos brasileños. Int. J. Morphol., 33(1):31-35, 2015.

RESUMEN: Entre los síndromes de compresión nerviosa en el antebrazo, debido a la presencia de arcos tendinosos de músculos relacionados al trayecto de los nervios que transcurren entre sus capas musculares, se encuentra el del nervio interóseo anterior (NIA). La presencia de variaciones musculares puede generar arcos que ocasionan compresión nerviosa. El objetivo fue establecer la incidencia y morfología del músculo accesorio del flexor largo del pulgar (AFLP) y la relación de éste con el NIA o sus ramos motores. Se utilizaron 30 antebrazos, de cadáveres formolizados de individuos adultos brasileños. El punto de referencia seleccionado para las mediciones fue el epicóndilo medial del humero (EMH). Se observó que tres miembros superiores presentaron el AFLP (10\%), en todos ellos se originó en el EMH para terminar insertándose en el tendón del músculo flexor largo del pulgar (FLP). El vientre muscular presentó aspecto fusiforme, el cual tenía un tendón que formó un arco con el FLP. En uno de los casos, este arco se relacionó con el paso del NIA; en los otros dos, lo hizo con los ramos motores que se originan del NIA. Al diagnosticar los síndromes generados por compresión nerviosa se debe considerar la presencia del AFLP, el cual, potencialmente se puede relacionar con el trayecto del NIA o de sus ramos.

PALABRAS CLAVE: Anatomía; Variaciones musculares; Cabeza accesoria del músculo flexor largo del pulgar; Nervio interóseo anterior; Síndrome de compresión.

\section{INTRODUCCIÓN}

Los músculos del miembro superior se desarrollan en base a la necesidad de realizar actividades prensiles por sobre un rol de apoyo del peso corporal. Durante este desarrollo de habilidades los diferentes músculos del antebrazo participan, tanto en la movilidad del complejo articular del codo, como también en la génesis de los más diversos movimientos de la mano.

La importancia de conocer en detalle la inervación de los diversos músculos del antebrazo hace necesario también describir los posibles sitios de compresión de los nervios que transcurren por esta parte del miembro superior. Este conocimiento permitiría inferir la forma de presentación de los diversos síndromes de atrapamiento nervioso en el antebrazo, descripción que parte de la base del conocimiento existente sobre la presencia de arcos fibrosos que diversos músculos del antebrazo ofrecen al trayecto de nervios.

El nervio mediano no emite ramos directos a los músculos profundos del compartimiento anterior del antebrazo, en su reemplazo, el encargado de dicha inervación en forma exclusiva es el nervio interóseo anterior (NIA), quien se desprende de la cara posterior del nervio mediano, en algunas oportunidades, a la altura del músculo pronador redondo, y en otras, cuando el nervio mediano pasa bajo el arco que forman las cabezas de origen del músculo flexor superficial de los dedos. En su trayecto el NIA entrega una serie de ramos que abordan en su longitud a los músculos flexor largo del pulgar, pronador cuadrado y la mitad lateral del flexor profundo de los dedos (Rauber, 1898; Tountas \& Bergman, 1993; Khale et al., 1994;

\footnotetext{
* Universidad Andres Bello, Sede Viña del Mar, Viña del Mar, Chile.

** Programa de Magíster en Ciencias, mención, Morfología, Universidad de La Frontera, Temuco, Chile.

*** Facultad de Medicina, Universidad de La Frontera, Temuco, Chile.

***** Universidade Estadual de Ciencias da Saude de Alagoas, Maceió, Brasil.
} 
Williams et al., 1998; Moore \& Dalley, 2002; Schünke et al., 2005; Leversedge et al., 2010; Pró, 2012; Llusá Pérez et al., 2013).

La existencia de variantes musculares en el trayecto del NIA puede generar la presencia de otros arcos que ocasionen compresión de este nervio, lo que puede desarrollar una debilidad de la pinza digital por afección del músculo flexor largo del pulgar y del vientre que el músculo flexor profundo de los dedos posee para el dedo índice.

Para Dellon \& Mackinnon (1987) y Pai et al. (2008), este fascículo accesorio que se puede originar desde el proceso coronoides de la ulna o desde el epicóndilo medial humeral está presente en un $46 \%$ de los miembros superiores. Esto coincide con la planteada por Llusá Pérez et al., quienes agregaron que el tendón de este fascículo se suma al tercio medio de la longitud del músculo flexor largo del pulgar y en su trayecto se dispone ventral al nervio interóseo anterior. La presencia de este músculo mencionada por estos autores es un poco menor a la encontrada por Oh et al., (2000) quienes observaron en una muestra de cadáveres asiáticos a este fascículo en un $66,7 \%$ y a Uyaroglu et al. (2006) quienes estudiando 26 cadáveres turcos lo encontraron en $51,9 \%$ de los casos.

Ante estos hallazgos surge la necesidad de establecer la frecuencia de este fascículo accesorio para el músculo flexor largo del pulgar en muestras que representen población sudamericana. De esta manera se obtendrá datos concretos de la presencia de esta variante muscular y su real impacto en síndromes de atrapamiento del NIA.

\section{MATERIAL Y MÉTODO}

En este estudio se utilizaron 30 antebrazos, de individuos adultos Brasileños, quienes pertenecen a la Facultad de Medicina de la Universidad Estadual de Ciencias da Saúde de Alagoas, Maceió, Brasil. Estos cadáveres se encontraban fijados con una solución de formaldehído al 10\%. Este estudio se formuló bajo una metodología de carácter cuantitativo y descriptivo, para la cual se procedió a realizar una detallada disección del compartimento anterior del antebrazo con material quirúrgico ad-hoc.

El punto de referencia seleccionado para las mediciones realizadas fue el epicóndilo medial del humero y las mediciones obtenidas fueron realizadas mediante la utilización de un caliper digital marca Mitutoyo de 0,01 mm de precisión.

\section{RESULTADOS}

Del total de 30 miembros disecados, tres presentaron este fascículo accesorio del músculo flexor largo del pulgar. En estos casos, este fascículo se originó en el epicóndilo humeral medial para terminar insertándose en el tendón del músculo flexor largo del pulgar. Dentro de los datos biométricos se destaca que los vientres musculares presentaron un aspecto fusiforme con un ancho promedio de $6,17 \mathrm{~mm}$, y una longitud promedio de $117,18 \mathrm{~mm}$. De este vientre muscular se observó un tendón que presentó una longitud promedio de $24,44 \mathrm{~mm}$ (Figs. 1 y 2).

Respecto a su relación con los diversos nervios y ramos motores descritos, se pudo establecer que en uno de los casos este fascículo accesorio generó un arco que se vinculó con el paso del nervio interóseo anterior. En los otros dos casos este arco se relacionó con los ramos motores que el nervio interóseo entregó para los músculos flexor profundo de los dedos y pronador cuadrado (Figs. 1 y 2).

\section{DISCUSIÓN}

Dentro de las variaciones musculares del miembro superior, se encuentra un músculo asociado al flexor largo del pulgar, el que fue descrito en 1813 por Gantzer (Testut \& Latarjet, 1972; Leversedge et al.). Autores como Dellon \& Mackinnon, Jones et al. (1997), Shirali et al. (1998), Oh et al., Uyaroglu et al., El Domiaty et al. (2008), Pai et al., Leversedge et al. y Llusá Pérez et al., entre otros, han descrito la presencia de un fascículo accesorio para el músculo flexor largo del pulgar, el cual presenta una prevalencia de entre $30 \%$ y $66,7 \%$ de los casos. Esta cifra resulta superior a la que se presentó en nuestro estudio, ya que de un total de 30 miembros disecados, tres miembros superiores presentaron este fascículo accesorio (10\%).

Con respecto al origen de esta variante muscular, los tres casos encontrados presentaron un origen en el epicóndilo medial del humero, cifra que se aleja de los $10,4 \%$ y $18,5 \%$ que Oh et al. y Uyaroglu et al., describieron en sus respectivos estudios, ya que se originaban principalmente del proceso coronoides de la ulna. Sin embargo, Mahakkanukrauh et al. (2004), informaron que en su serie estudiada en individuos Tailandeses (120 casos) el $74,5 \%$ se originó en el epicóndilo medial del húmero.

Los tres casos hallados, se presentaron de forma unilateral, descripción que difiere al reporte de caso relatado por Eid \& Otsuky (2009), en el cual un individuo de origen 


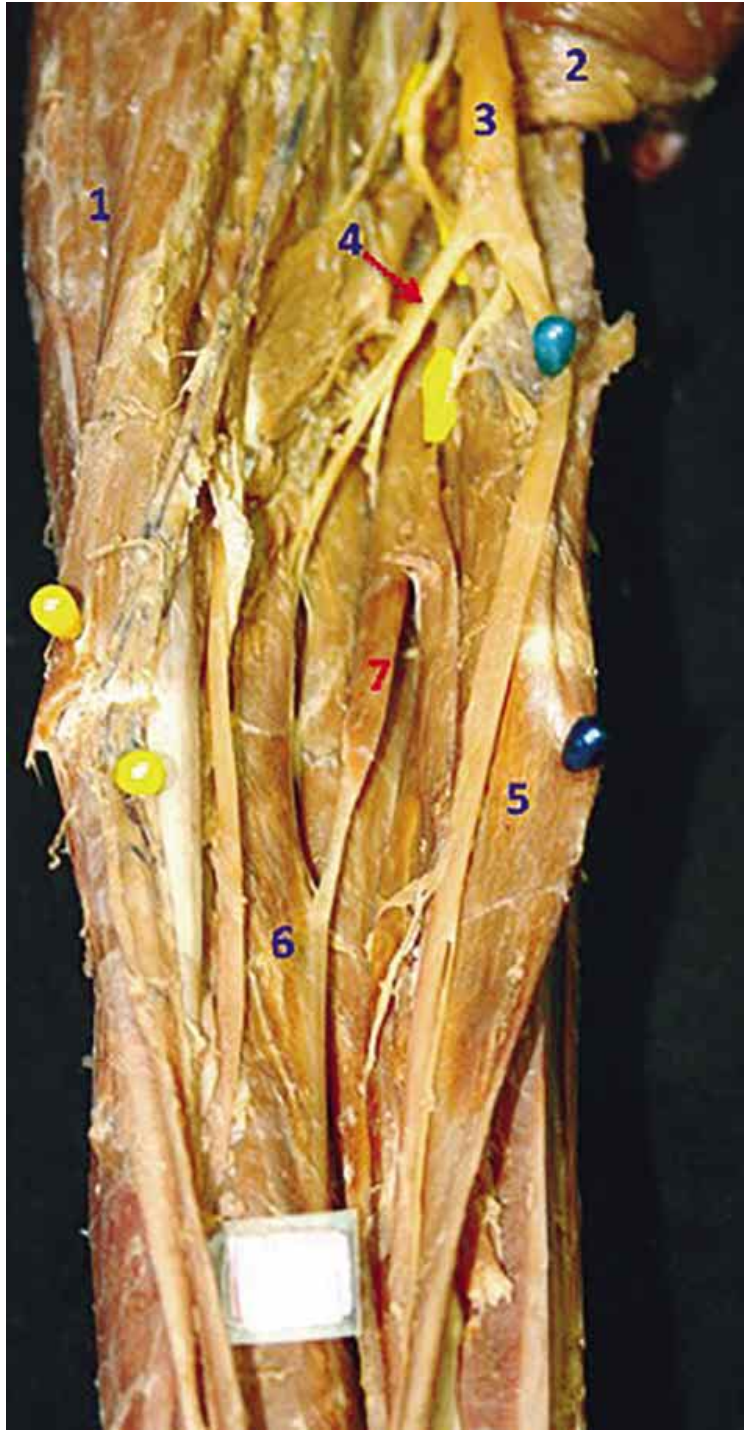

Fig. 1. Antebrazo donde se observan los elementos musculares y el nervio interóseo anterior. 1= Músculo Braquiorradial, 2= Músculo Pronador Redondo, 3= Nervio mediano, 4= Nervio interóseo anterior, 5= Músculo flexor superficial de los dedos, 6= Músculo Flexor largo del pulgar, 7= Fascículo accesorio del músculo flexor largo del

japonés presentó este fascículo de forma bilateral; la serie de Uyaroglu et al., arrojó bilateralidad en 10 de 26 individuos $(38,5 \%)$ y Jones et al. un $17,5 \%$. Un porcentaje mayor (75,3\% - 64/85) fue informado por Mahakkanukrauh et al.

Respecto a la morfología del fascículo accesorio para el músculo flexor largo del pulgar, Jones et al., señalaron que este fascículo, que observaron en un 30\% de los casos y cuyo tendón se sumó en más del 70\% de los casos al tercio proximal del flexor largo del pulgar, presentó diversas características de su vientre muscular, siendo de poco espesor

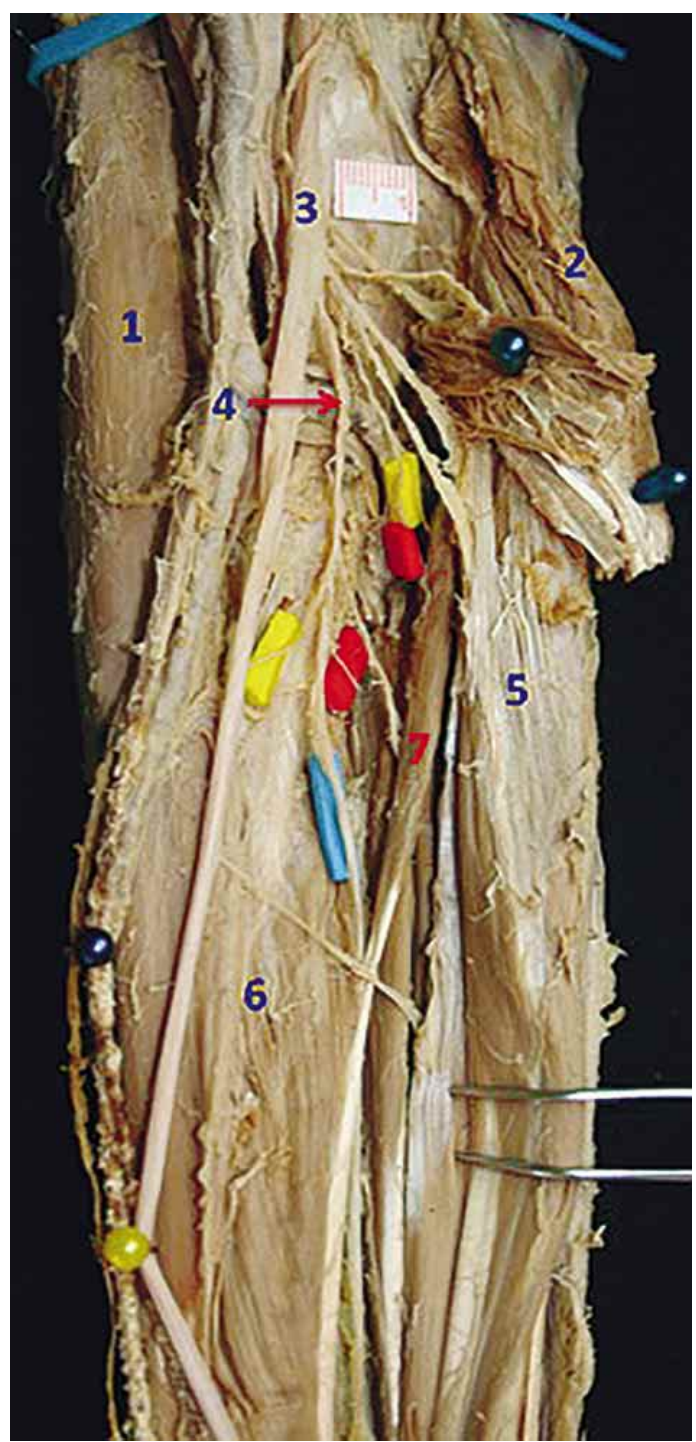

Fig. 2. Antebrazo donde se observan los elementos musculares y el nervio interóseo anterior. $1=$ Músculo Braquiorradial, 2= Músculo Pronador Redondo, 3= Nervio mediano, $4=$ Nervio interóseo anterior, 5= Músculo flexor superficial de los dedos, 6= Músculo Flexor largo del pulgar, 7= Fascículo accesorio del músculo flexor largo del pulgar.

en $42,9 \%$, voluminoso en $28,6 \%$, triangular en $15,3 \%$ y fusiforme en un $14,2 \%$, esta última considerada por El Domiaty et al., en un 53,8\% de su muestra. Al comparar estos datos con los de nuestro estudio, se observó que en los 3 casos el vientre muscular presentó aspecto fusiforme, y tuvieron mediciones similares al reporte de caso de Campos et al. (2009), en donde ambos estudios presentaron un vientre muscular con un ancho promedio de $6,17 \mathrm{~mm}$, y una longitud promedio de 117,18 mm. En una muestra de un grupo de individuos egipcios, El Domiaty et al. registraron una longitud total de $74,66 \mathrm{~mm}$. 
En relación a la presencia de arcos fibrosos que potencialmente pueden comprimir el nervio interóseo anterior, se pudo establecer que en uno de nuestros casos se presentó un arco que se relacionó con el paso del nervio interóseo anterior. En los casos restantes este arco se dispuso próximo a los ramos motores que el nervio interóseo entregó para los músculos flexor profundo de los dedos y pronador cuadrado. La relación entre este músculo accesorio y el nervio interóseo anterior fue reportada por Uyaroglu et al., quienes indicaron que el nervio se localizó posterior en $55,6 \%$ y posterolateral en $29,6 \%$ de los casos. Solo en un caso éste fue anterior al músculo mencionado.

Estos antecedentes resultan importantes si se trata de diferenciar los cuadros de compresión nerviosa, ya que en caso de que el paciente sufra una compresión del ner- vio interóseo anterior se genera el síndrome de Kiloh-Nevin el cual presenta una debilidad de la pinza digital por afección del flexor largo del pulgar y el vientre que el músculo flexor profundo de los dedos posee para el dedo índice. Uno de los signos clínicos que demuestra este debilidad, esta caracterizado por la incapacidad de realizar una letra "O" entre los dedos índice y el pulgar.

En la serie presentada, el fascículo accesorio para el músculo flexor largo del pulgar tuvo una incidencia baja respecto a otros estudios, pero del mismo modo puede formar arcos que podrían atrapar al nervio interóseo anterior. Al relacionar este hallazgo con la forma de presentación de los síndromes de compresión en el antebrazo, la presencia de ésta y otras variantes musculares pueden dificultar el correcto diagnóstico de estos síndromes.

RIVEROS, A.; OLAVE, E. \& SOUSA-RODRIGUES, C. Anatomical study of the accessory head of the flexor pollicis longus muscle and its relationship to the anterior interosseous nerve in Brazilian individuals. Int. J. Morphol., 33(1):31-35, 2015.

SUMMARY: Between the syndromes of nerve compression in the forearm, due to the presence of the tendinous arches related to the nerves that pass between its muscle layers, is the anterior interosseous nerve (AIN). The presence of muscular variations can generate arches that cause nerve compression. The aim of our study was to establish the incidence and morphology of the accessory head of flexor pollicis longus muscle (AFPLm) and its relationship with AIN or its branches. Thirty forearms of formalized corpses of adult Brazilians were used. The selected landmark for measurements was the medial epicondyle of the humerus (MEH). It was noted that three upper limbs presented the AFPLm (10\%), originated from the MEH to end inserted into the tendon of the flexor pollicis longus muscle. The AFPLm showed fusiform appearance, which had a tendon that formed an arch with the flexor pollicis longus muscle. In one case, this arch was associated with the passage of AIN. In the other two, this arch was related to motor branches originating from the AIN. The diagnosis of nerve compression syndromes should consider the presence of AFLPm, which potentially can be related to the course of the AIN or its branches.

KEY WORDS: Anatomy; Muscle variations; Head accessory flexor pollicis longus muscle; Anterior interosseous nerve; Compression syndrome.

\section{REFERENCIAS BIBLIOGRÁFICAS}

Campos, D.; Nazer, M. B. \& Bartholdy, L. M. Anatomical variation of the accessory muscle of the forearm (Gantzer's muscles) and his relationship with the median nerve: a case report in human. Braz. J. Morphol. Sci., 26(1):39-41, 2009.

Dellon, A. L. \& Mackinnon, S. E. Musculoaponeurotic variations along the course of the median nerve in the proximal forearm. J. Hand. Surg. Br., 12(3):359-63, 1987.

Eid, N. \& Otsuki, Y. A case of double Gantzer's muscle and its possible role in nerve entrapment. Clin. Anat., 22(8):881-2, 2009.

El Domiaty, M. A.; Zoair, M. M. \& Sheta, A. A. The prevalence of accessory heads of the flexor pollicis longus and the flexor digitorum profundus muscles in Egyptians and their relations to median and anterior interosseous nerves. Folia Morphol. (Warsz.), 67(1):63-71, 2008.
Jones, M.; Abrahams, P. H.; Sañudo, J. R. \& Campillo, M. Incidence and morphology of accessory heads of flexor pollicis longus and flexor digitorum profundus (Gantzer's muscles). J. Anat., 191(Pt. 3):451-5, 1997.

Khale, W.; Leonhardt, H. \& Platzer, W. Atlas de Anatomía. Vol 3. 5a ed. Barcelona, Omega, 1994. pp.375.

Leversedge, F. J.; Goldfarb, C. A. \& Boyer, M. I. A pocketbook Manual of hand and upper extremity anatomy: Primus manus. Philadelphia, Lippincott Williams \& Wilkins, 2010. pp.256.

Llusá Pérez, M.; Palazzi Coll, S. \& Valer Tito, A. Anatomía quirúrgica del plexo braquial y nervios periféricos de la extremidad superior. Buenos Aires, Médica Panamericana, 2013. pp.374.

Mahakkanukrauh, P.; Surin, P.; Ongkana, N.; Sethadavit, M. \& 
RIVEROS, A.; OLAVE, E. \& SOUSA-RODRIGUES, C. Estudio anatómico del fascículo accesorio del músculo flexor largo del pulgar y su relación con el nervio interóseo anterior en individuos brasileños. Int. J. Morphol., 33(1):31-35, 2015.

Vaidhayakarn, P. Prevalence of accessory head of flexor pollicis longus muscle and its relation to anterior interosseous nerve in Thai population. Clin. Anat., 17(8):631-5, 2004.

Moore, K. \& Dalley, A. Anatomía con orientación clínica. $4^{\mathrm{a}}$ ed. Buenos Aires, Médica Panamericana, 2002. pp.1185.

Oh, C. S.; Chung, I. H. \& Koh, K. S. Anatomical study of the accessory head of the flexor pollicis longus and the anterior interosseous nerve in Asians. Clin. Anat., 13(6):434-8. 2000.

Pai, M. M.; Nayak, S. R.; Krishnamurthy, A.; Vadgaonkar, R.; Prabhu, L.; Ranade, A. V.; Janardhan, J. \& Rai, R. The accessory heads of flexor pollicis longus and flexor digitorum profundus: Incidence and morphology. Clin. Anat., 21(3):2528, 2008.

Pró, E. A. Anatomía Clínica. Buenos Aires, Médica Panamericana, 2012. pp.992.

Rauber, A. Anatomie des Menschen. 5a ed. Leipzig, Verlag von Arthur Georgi, 1898. Vol 2. pp.882.

Schünke, M.; Schulte, E. \& Schumacher, U. Prometheus: Texto y Atlas de Anatomia. Buenos Aires, Médica Panamericana, 2005. Vol. 1. pp.539.

Shirali, S.; Hanson, M.; Branovacki, G. \& Gonzalez, M. The flexor pollicis longus and its relation to the anterior and posterior interosseous nerves. J. Hand Surg., 23(2):170-2, 1998.

Tountas, C. \& Bergman, R. Anatomic variations of the upper extremity. New York, Churchill Livingstone, 1993. pp.286.

Testut, L. \& Latarjet, A. Tratado de anatomía humana. 9a. ed. Barcelona, Salvat, 1972. Vol. 1. pp.1198.

Uyaroglu, F. G.; Kayalioglu, G. \& Erturk, M. Incidence and morphology of the accessory head of the flexor pollicis longus muscle (Gantzer`s muscle) in a Turkish population. Neurosciences (Riyadh), 11(3):171-4. 2006.

Williams, P. L.; Bannister, L. H.; Berry, M. M.; Collins, P.; Dyson, M.; Dussek, J. E. \& Ferguson, M. W. J. Anatomía de Gray. Bases Anatómicas de la Medicina y la Cirugía. $38^{\mathrm{a}}$ ed. Madrid, Churchill Livingstone-Harcourt Brace, 1998. Vol 2.

\author{
Dirección para Correspondencia: \\ Dr. Enrique Olave \\ Facultad de Medicina \\ Universidad de La Frontera \\ Casilla 54-D, Temuco \\ CHILE
}

Email: enrique.olave@ufrontera.cl

Recibido : 12-08-2014

Aceptado: 22-11-2014 